\title{
Contracts in informed-principal problems with moral hazard
}

\author{
Nicholas C Bedard*
}

January 20, 2016

\begin{abstract}
In many cases, an employer has private information about the potential productivity of a worker, who in turn has private information about the effort she exerts on the job. Much of the analysis of this environment in the literature restricts the employer to offer contracts that depend only on observable outcomes (e.g. profit). This paper studies the advantages to the employer of offering the worker a set of potential contracts from which the employer will choose after the worker has accepted the offer, so called menucontracts. Specifically, in a two-state principal-agent problem with moral hazard, I show when the principal can obtain strictly higher expected payoffs than the restricted contracts of the literature by offering a menu-contract.
\end{abstract}

\section{Introduction}

In many cases, an employer has private information about the potential productivity of a worker, who in turn has private information about the effort she exerts on the job. For example, a law firm may know the likelihood of winning a trial but cannot observe the effort the attorney exerts for the case. In such situations, the employer needs to design a contract that releases the right information at the right time to optimally exploit the information asymmetry.

*ESEI Center for Market Design, University of Zürich, Schönberggasse 1, CH-8001. Phone: +41 44634 37 43. Email: nick.bedard85@gmail.com. The author gratefully acknowledges financial support from the Social Sciences and Humanities Research Council of Canada, the Ontario Graduate Scholarship, and the European Research Council (ERC Advanced Investigator Grant, ESEI-249433). 
A number of papers have analyzed the value of information in such environments; see for example Beaudry (1994); Chade and Silvers (2002); ${ }^{1}$ Kaya (2010); Martimort et al. (2010); and Silvers (2012); Inderst (2001) also studies this environment but does not consider the value of the principal's information. These papers, however, restrict the set of contracts the principal can propose to the set of point-contracts; that is, contracts that depend only on observable outcomes, specifically, the success or failure of the project. In this article I show how this restriction affects the principal's equilibrium payoffs relative to contracts that allow the principal to condition contracts on her own private information as well. Such contracts are called menu-contracts in the literature since they take the form of a list of contracts that is offered to the agent from which the principal chooses after the agent has accepted.

Formally, the model is of an informed principal problem with moral hazard. The principal cannot observe if the agent has exerted effort but does observe the outcome of the task: it can be either a failure or a success. The task can be either productive or unproductive: exerting effort is more likely to lead to success in the productive task than in the unproductive task. The principal knows if the task is productive but the agent does not.

When the principal is restricted to offer only point contracts she is subject to the agent's arbitrary off-path posterior beliefs. For example, very inefficient point-contracts can be supported in equilibrium by specifying that the agent's off-path beliefs put probability one on the principal being the worst type (from the agent's point of view). I characterize the lower bound equilibrium payoff for the principal when she can offer menu-contracts and show that it is always strictly higher than the lower bound when the principal is restricted to offer point-contracts, whenever a separating equilibrium exists in point-contracts. In particular, when it exists, the least-cost-separating contract is the best point-contract for the principal but achieves the lower bound equilibrium menu-contract payoff. ${ }^{2}$ This lower bound menu-contract is belief free: it is acceptable to the agent regardless of her beliefs

\footnotetext{
${ }^{1}$ Chade and Silvers (2002) do consider more general contracts as a robustness check but mainly focus on point-contracts. See below for more details.

${ }^{2}$ When no separating equilibrium exists, the set of point-contract equilibrium payoffs may coincide with the set of menu-contract equilibrium payoffs.
} 
about the principal's type and therefore not susceptible to punishing off-path beliefs. In this way, menu-contracts help to refine the set of equilibria without needing to design ad-hoc selection criteria.

Finally, I characterize environments in which separating menu-contracts exist that are strictly better for the principal than any separating point-contract. Beaudry (1994), Chade and Silvers (2002), and Silvers (2012) show that the best separating point-contract cedes rents to the agent ex ante in the form of higher base wages (i.e. a flatter wage profile). I construct a menu-contract that eliminates these signalling costs and thus reduces the principal's wage bill. In particular, I show that if and only if the least-cost-separating equilibrium pointcontract is strictly above the minimum wage allowed, there exists a menu-contract that is strictly less expensive for the principal. If there is no minimum wage (i.e. the agent has unlimited liability), then the principal should never cede rents to the agent ex ante. Essentially, the principal can use menu-contracts to squeeze more rent from the agent by eliminating the slack in the agent's ex ante individual rationality constraint and transferring it across states in a way that preserves her own incentive compatibility.

\section{$1.1 \quad$ Literature}

The general problem of the privately informed principal is developed in Myerson (1983); in specialized environments with no moral hazard, Maskin and Tirole $(1990,1992)$ characterize the equilibria of the model and derive their properties. In particular, Maskin and Tirole (1992) characterize a contract that earns the principal her lower bound equilibrium payoff. As in this paper, Segal and Whinston (2003) apply Maskin and Tirole's methodology to reduce the indeterminacy of equilibria in a class of bilateral contracting problems. Their work differs from the current paper in that the private information of the principal is her profile of trades with other agents, and therefore is endogenous in their model.

A number of papers have studied the informed principal problem with moral hazard: for example see Beaudry (1994), Chade and Silvers (2002), Kaya (2010), Martimort et al. 
(2010), Silvers (2012), Inderst (2001) and Wagner et al. (2015). To my knowledge, there has not been a characterization of the equilibria in this model without restricting the principal's contract choice. Notably, Chade and Silvers (2002) show that the equilibrium in their result, the least-cost-separating equilibrium, can be achieved via a menu-contract. I show that the principal can never do worse than under this menu-contract equilibrium and, depending on the parameters of the model, can do strictly better with another menu-contract. Wagner et al. (2015) describe a class of informed principal problems with moral hazard wherein each type of principal earns her first-best payoff. ${ }^{3}$ In such cases, menu-contracts cannot improve on the best point-contract but can eliminate some inefficient point-contract equilibria.

\section{Model}

\subsection{Preferences and Technologies}

The agent is (weakly) risk averse and maximizes expected utility. Her payoff over wage income, $w \in[\underline{w}, \infty)$, and effort, $a \in A:=\left\{a_{1}, a_{2}\right\} \subset \mathbb{R}_{+}^{2}$, is given by $U(w)-a$ with $U^{\prime}(w)>0$ and $U^{\prime \prime}(w) \leq 0$. Assume that $0 \leq a_{1}<a_{2}<\infty$ and $\underline{w} \in \mathbb{R} \cup\{-\infty\}$. Note that the lower bound on the set of wage offers implies limited liability for the agent if $\underline{w}>-\infty$. Let $h:=U^{-1}$ denote the inverse of $U$.

The effort chosen induces a conditional probability distribution over the set of possible outcomes $Q:=\left\{q_{s}, q_{f}\right\} \subset \mathbb{R}_{+}^{2}$ where $0 \leq q_{f}<q_{s}<\infty$ (the subscript $f$ denotes failure while $s$ denotes success). If the principal pays the agent wage $w$ and outcome $q_{n}$ is realized her payoff is $q_{n}-w$.

The principal is a risk neutral expected profit maximizer who needs to hire the agent to complete a task. Task productivity can either be high or low. Each type of task is associated with a set of conditional probability distributions that determines the probability

\footnotetext{
${ }^{3}$ Their analysis allows the principal to offer menu-contracts but the same payoffs can be achieved using point-contracts.
} 
of the task being successful given a particular effort level. The task of type $i \in\{H, L\}$ has probability distribution $\Pi^{i}:=\left(\boldsymbol{\pi}^{i}\left(a_{1}\right), \boldsymbol{\pi}^{i}\left(a_{2}\right)\right)$ where $\boldsymbol{\pi}^{i}(a):=\left(\pi_{s}^{i}(a), \pi_{f}^{i}(a)\right)$ denotes the conditional probability distribution across $Q$ when the effort is $a \in A$. The type- $H$ task is more productive than the type- $L$ task: $\pi_{s}^{H}\left(a_{2}\right)>\pi_{s}^{L}\left(a_{2}\right)$ and $\pi_{s}^{H}\left(a_{1}\right) \geq \pi_{s}^{L}\left(a_{1}\right)$; and higher effort is more productive: $\pi_{s}^{i}\left(a_{2}\right)>\pi_{s}^{i}\left(a_{1}\right)$ for $i \in\{H, L\}$. For example, if the attorney from the Introduction exerts a unit of effort on an easy case she is more likely to win than if she exerted the same unit of effort on a difficult case and, for any type of case, she is more likely to win if she exerts effort. Let $\lambda \in(0,1)$ be the common prior probability that the task is of high productivity; i.e. has conditional probability distribution $\Pi^{H}$. I refer to a principal who has task type $i$ as a type- $i$ principal. Revenue is assumed to be sufficiently large that there always exists at least one equilibrium in which the type- $H$ principal hires an agent.

\subsection{Contracts}

A point-contract is a set of payments $\mathbf{w}=\left(w_{s}, w_{f}\right) \in[\underline{w}, \infty)^{2}$ from the principal to the agent and an effort level $a \in A$ such that the principal pays the agent $w_{n}$ when outcome $q_{n}$ is realized for $n \in\{s, f\}$ and suggests the agent take effort level $a$. Let $\mathbf{U}(\mathbf{w})=\left(U\left(w_{f}\right), U\left(w_{s}\right)\right)$ and $v^{i}(\mathbf{w}, a):=\boldsymbol{\pi}^{i}(a) \cdot(\mathbf{q}-\mathbf{w}){ }^{4}$

A menu-contract is direct revelation mechanism that specifies a pair of point-contracts $\left(\mathbf{w}^{i}, a^{i}\right)_{i \in\{H, L\}}$ such that $\mathbf{w}^{i} \in[\underline{w}, \infty)^{2}$ and $a^{i} \in A$ for all $i \in\{H, L\}$. The principal chooses from this set after the agent has accepted the offer. ${ }^{5}$

\subsection{Information}

The principal knows her type but the agent does not. Let $\boldsymbol{\rho}:=\left(\rho^{H}, \rho^{L}\right)$ denote the agent's posterior belief over the type of the principal after observing the proposed contract. Further, let $\mathbf{p}(a ; \boldsymbol{\rho})=\left(p_{s}(a ; \boldsymbol{\rho}), p_{f}(a ; \boldsymbol{\rho})\right):=\rho^{H} \boldsymbol{\pi}^{H}(a)+\rho^{L} \boldsymbol{\pi}^{L}(a)$ denote the agent's expected prob-

\footnotetext{
${ }^{4}$ Where $\cdot$ denotes the inner product operator.

${ }^{5}$ The formal timing of the game is outlined in Section 2.4.
} 
ability distribution over $Q$ conditional on having chosen effort $a \in A$ where $p_{n}(a ; \boldsymbol{\rho})$ is her belief that outcome $q_{n}$ will occur conditional on having chosen effort $a \in A$ for $n \in\{s, f\}$.

\subsection{Timing}

The timing of the game is as follows:

$\begin{array}{ccccc}1 & 2 & 3 & 4 & 5 \\ \begin{array}{c}\text { Realization of } \\ \text { information }\end{array} & \begin{array}{c}\text { Contract } \\ \text { offer }\end{array} & \begin{array}{c}\text { Agent } \\ \text { response }\end{array} & \text { Contract choice } & \begin{array}{c}\text { Realization of outcome } \\ \text { and implementation }\end{array}\end{array}$

In stage 1, nature chooses the principal's type and this is observed by the principal. In stage 2 , the principal offers the agent a contract. The agent updates her priors based on the stage 2 offer and accepts or rejects the contract in stage 3 . If the agent rejects the contract, the principal receives zero utility and the agent receives utility $\bar{U}$. If only point-contracts are available, the game skips to stage 5: the agent chooses her effort level, the outcome is realized and the corresponding wage is paid. If menu-contracts are available, in stage 4 the principal chooses from the menu-contracts and stage 5 follows as before.

\subsection{Constraints}

\subsubsection{Point-contracts}

A point-contract $\left(\mathbf{w}, a_{k}\right)$, for $k \in\{1,2\}$ is individually rational if

$$
\mathbf{p}\left(a_{k} ; \boldsymbol{\rho}\right) \cdot \mathbf{U}(\mathbf{w})-a_{k} \geq \bar{U}
$$

The contract is incentive compatible for the agent if, for $k=2$, the agent does not want to exert low effort

$$
\left(\mathbf{p}\left(a_{2} ; \boldsymbol{\rho}\right)-\mathbf{p}\left(a_{1} ; \boldsymbol{\rho}\right)\right) \cdot \mathbf{U}(\mathbf{w}) \geq a_{2}-a_{1}
$$

The upward constraint, which prevents her from taking high effort when the principal wants her to take low effort, will never bind in equilibrium. To see this, note that for any constant 
wage $\mathbf{w},\left(\mathbf{p}\left(a_{2} ; \boldsymbol{\rho}\right)-\mathbf{p}\left(a_{1} ; \boldsymbol{\rho}\right)\right) \cdot \mathbf{U}(\mathbf{w})=0 ;$ moreover, since $a_{1}-a_{2}<0, \mathbf{w}$ satisfies the upward incentive constraint for $k=1$. Finally, note that the optimal wage implementing low effort will be constant across $s$ and $f$ : since the agent is (weakly) risk averse, for any wage w, the constant wage equal to the certainty equivalent of $\mathbf{w}$ will be (weakly) cheaper for the risk neutral principal.

A pair of point-contracts $\left(\left(\mathbf{w}^{H}, a^{H}\right),\left(\mathbf{w}^{L}, a^{L}\right)\right)$ is incentive compatible for the principal if it satisfies for all $i, j \in\{H, L\}$

$$
\boldsymbol{\pi}^{i}\left(a^{i}\right) \cdot\left(\mathbf{q}-\mathbf{w}^{i}\right) \geq \boldsymbol{\pi}^{i}\left(a^{j}\right) \cdot\left(\mathbf{q}-\mathbf{w}^{j}\right)
$$

\subsubsection{Menu-contracts}

Myerson's inscrutability principle (Myerson (1983)) states that it is without loss of generality to assume that all types of the principal offer the same menu of contracts in equilibrium. ${ }^{6}$ This implies that a menu-contract need only satisfy the agent's individual rationality constraint ex ante. A menu-contract $\left(\left(\mathbf{w}^{H}, a^{H}\right),\left(\mathbf{w}^{L}, a^{L}\right)\right)$ is therefore individually rational if

$$
\lambda\left(\boldsymbol{\pi}^{H}\left(a^{H}\right) \cdot \mathbf{U}\left(\mathbf{w}^{H}\right)-a^{H}\right)+(1-\lambda)\left(\boldsymbol{\pi}^{L}\left(a^{L}\right) \cdot \mathbf{U}\left(\mathbf{w}^{L}\right)-a^{L}\right) \geq \bar{U} .
$$

A separating menu-contract (i.e. a menu-contract $\left(\mathbf{w}^{i}, a^{i}\right)_{i \in\{H, L\}}$ such that $\mathbf{w}^{H} \neq \mathbf{w}^{L}$ or $\left.a^{H} \neq a^{L}\right)$ is incentive compatible for the agent if $A I C(1,0)$ and $A I C(0,1)$ are satisfied for $i \in\{H, L\}$; that is, in a separating contract, the agent's incentive compatibility constraints for each principal type- $i$ must be satisfied given that the agent believes the principal's type is $i$ with probability one. A pooling menu-contract is incentive compatible for the agent if $\left(\mathbf{w}^{H}, a^{H}\right)=\left(\mathbf{w}^{L}, a^{L}\right)$ and it satisfies $A I C(\lambda, 1-\lambda)$. Finally, a menu-contract is incentive compatible for the principal if it satisfies $P I C(i, j)$ for $i, j \in\{H, L\}$.

\footnotetext{
${ }^{6}$ Thus, the agent cannot extract any information from the principal's offer; the principal is inscrutable at the time of the agent's acceptance decision.
} 


\section{Preliminary Analysis}

In this section I establish a number of benchmark equilibrium contracts and their payoffs. I first describe the contracts that would result if the principal's type were common knowledge; the payoff from these contracts is called the public information benchmark. It is the firstbest ex ante payoff for the principal. Further, I describe the lowest payoffs supported by an equilibrium in the game where only point-contracts can be offered and the game where menu-contracts can be offered.

To focus on interesting cases, the following assumption is maintained throughout.

Assumption $1 \pi_{s}^{H}\left(a_{2}\right)-\pi_{s}^{H}\left(a_{1}\right)>\pi_{s}^{L}\left(a_{2}\right)-\pi_{s}^{L}\left(a_{1}\right)$.

This assumption states that the marginal product of effort is larger when the principal's type is $H$. It is a sufficient condition for the existence of a separating equilibrium, as long as the type- $H$ principal's first-best effort level is $a_{2}$.

\subsection{The Public Information Benchmark}

Suppose the principal's type is public information. For each effort level $a \in A$, the principal of type $i \in\{H, L\}$ chooses $\mathbf{w}$ to minimize $\boldsymbol{\pi}^{i}(a) \cdot \mathbf{w}$ subject to $A I C(\boldsymbol{\rho})$ and $I R(\boldsymbol{\rho})$ where $\rho^{i}=1$. Denote the solution to this problem by $\mathbf{w}^{* i}(a)$. The principal then chooses the effort $a$ that maximizes $\boldsymbol{\pi}^{i}(a) \cdot\left(\mathbf{q}-\mathbf{w}^{* i}(a)\right)$. Denote this effort by $a^{* i}$. Let $\mathbf{w}^{* i}:=\mathbf{w}^{* i}\left(a^{* i}\right)$ and $v^{* i}:=\boldsymbol{\pi}_{i}\left(a^{* i}\right) \cdot\left(\mathbf{q}-\mathbf{w}^{* i}\right)$.

\subsection{Lower Bound Equilibrium Point-Contract Payoff}

The lower bound point-contract payoff is obtained when the principal chooses $(\mathbf{w}, a)$ to maximize $\boldsymbol{\pi}^{i}(a) \cdot(\mathbf{q}-\mathbf{w})$ subject to $A I C(0,1)$ and $I R(0,1)$; that is, both principal types choose a contract to maximize profit subject to constraints that assume the agent puts probability one on the event that the principal's type is $L .^{7}$ This equilibrium is supported

\footnotetext{
${ }^{7}$ If the choice of effort is the same for both principal types, each will offer $\left(\mathbf{w}^{* L}, a^{* L}\right)$. In some cases, the high type principal may find it profitable to induce effort $a_{2}$ while the low type principal prefers $a_{1}$;
} 
by agent beliefs that put probability one on the principal being type- $L$ whenever a deviation is observed. To see that this is an equilibrium, note that any incentive compatible, individually rational deviation contract must, by definition, not be more profitable for the principal given the agents belief.

\subsection{Lower Bound Equilibrium Menu-Contract Payoff}

In the spirit of Maskin and Tirole (1992) a menu-contract $\left(\hat{\mathbf{w}}^{i}, \hat{a}^{i}\right)_{i \in\{H, L\}}$ is called a RothchildStiglitz-Wilson (RSW) contract if, for each type- $i$ principal, it maximizes $v^{i}(\mathbf{w}, a)$ subject to $I R(1,0), I R(0,1), A I C(1,0), A I C(0,1)$ and $P I C(i, j)$ for $i, j \in\{H, L\} .^{8}$ Let $\left(\hat{\mathbf{w}}^{i}, \hat{a}^{i}\right)_{i \in\{H, L\}}$ denote the RSW menu-contract and $\hat{v}^{i}:=v^{i}\left(\hat{\mathbf{w}}^{i}, \hat{a}^{i}\right)$ denote the RSW payoff. ${ }^{9}$

When menu-contracts are allowed, the RSW problem generates lower bound payoffs for the type- $i$ principal since the agent will accept any RSW contract regardless of her belief about the type of the principal. ${ }^{10}$ To see this, note first that the RSW problem for the type- $i$ principal specifies an entire menu: a contract for each principal type $j \in\{H, L\}$. This menu must be incentive compatible for every principal type $j \in\{H, L\}$, not just type- $i$. Finally, this menu-contract must guarantee the agent her reservation payoff ex post and induce the appropriate effort level regardless of the principal's type. Thus, the agent will always accept an RSW contract and exert the correct amount of effort. In any equilibrium in which the type- $i$ principal obtains a payoff less than $\hat{v}^{i}$, she can therefore deviate to her RSW contract to obtain $\hat{v}^{i}$. Figure 1 displays RSW contracts for risk neutral agents. ${ }^{11}$

then the type- $L$ principal offers $\left(\mathbf{w}^{* L}, a^{* L}\right)$ and the type- $H$ principal offers $\left(\mathbf{w}^{* L}\left(a_{2}\right), a_{2}\right)$, that is, the type- $L$ optimal wage when effort is fixed at $a_{2}$. Notice that if a wage profile satisfies $A I C(0,1)$ then it also satisfies $A I C(1,0)$, since $\pi_{s}^{H}\left(a_{2}\right)-\pi_{s}^{H}\left(a_{1}\right)>\pi_{s}^{L}\left(a_{2}\right)-\pi_{s}^{L}\left(a_{1}\right)$. Moreover, for any wage profile $\mathbf{w}$ that satisfies $A I C(\boldsymbol{\rho})$ for any $\boldsymbol{\rho}$, it must be that $w_{s}>w_{f}$, since $a_{2}>a_{1}$. Therefore, since $\pi^{H}\left(a_{2}\right)>\pi^{L}\left(a_{2}\right)$, if a wage profile satisfies $\operatorname{IR}(0,1)$ then it also satisfies $\operatorname{IR}(1,0)$.

${ }^{8}$ Rothchild-Stiglitz-Wilson is a reference to the similar least-cost-separating contracts developed in the insurance models of Rothschild and Stiglitz (1976) and Wilson (1977).

${ }^{9}$ The RSW menu-contract always exists and is unique.

${ }^{10}$ In terms of Myerson (1983), any feasible solution to the RSW problem is safe. The RSW menu for the type- $i$ principal is her best safe menu.

${ }^{11}$ Due to the linearity of the principal's indifference curves (in particular, the fact that they posses the single crossing property) computing the RSW contract can be simplified, as I show in Lemma 1 in the Appendix. This lemma extends Proposition 2 in Maskin and Tirole (1992). 


\section{The Deficiency of Point-Contracts}

The RSW equilibrium contract for the type- $i$ principal earns her the lower bound menucontract equilibrium. The RSW payoff can be obtained using point-contracts: if separating equilibria exist, it is the least-cost separating equilibrium. On the other hand, if Assumption 1 is satisfied and the high type principal wants to induce high effort in equilibrium, the RSW/least-cost-separating equilibrium is strictly better than the lower bound point-contract. $^{12}$ Essentially, whenever the principal is able to separate, there will be a point-contract equilibrium payoff that is strictly worse than any menu-contract equilibrium payoff.

One can show that if $\lambda$ is close to one, both of the principal's types prefer a pooling contract to the least-cost-separating contract. Since the pooling contract is implementable in point-contracts, this implies that the best point-contract is preferred to the worst menucontract. On the other hand, if $\lambda$ is small, the type- $H$ principal prefers the least-costseparating contract while the type- $L$ principal prefers a pooling contract. ${ }^{13}$ I now establish when a separating menu-contract equilibrium exists that is more profitable than the leastcost-separating contract for the principal.

\subsection{Separating Equilibria}

Let $\mathscr{P}^{S}$ and $\mathscr{M}^{S}$ be the sets of ex ante equilibrium payoffs for the principal that can be earned via separating point-contracts and separating menu-contracts respectively. Our next result describes when $\mathscr{M}^{S}$ is strictly higher than $\mathscr{P}^{S}$ (except at the RSW payoff). I first make the following assumptions.

Assumption 2 (a) $\boldsymbol{\pi}^{H}\left(\hat{a}^{H}\right) \cdot U\left(\hat{\mathbf{w}}^{H}\right)-\hat{a}^{H}>\bar{U}$; and $(b) v^{* L}<v^{L}\left(\mathbf{w}^{* H}, a^{* H}\right)$.

\footnotetext{
${ }^{12}$ Otherwise, one can show that menu- and point- contract equilibrium payoffs coincide.

${ }^{13}$ In this case, the pooling contract is not an equilibrium in menu-contracts because the type- $H$ principal gets a payoff that is lower than $\hat{v}^{H}$. Ex ante, the pooling contract may give a higher payoff than the leastcost-separating contract. This demonstrates that a menu-contract is not a generalization of a point-contract.
} 
Part (a) of the assumption states that the agent earns more than her reservation utility when the principal is type $H$ under the least-cost-separating equilibrium. This holds, for example, when the relative likelihood of a higher outcome to a lower outcome is increasing in the effort, i.e. $\frac{\pi_{s}^{i}\left(a_{1}\right)}{\pi_{f}^{i}\left(a_{1}\right)}<\frac{\pi_{s}^{i}\left(a_{2}\right)}{\pi_{f}^{i}\left(a_{2}\right)}$, and $w_{s}^{* H}>w_{f}^{* H}$. Part (b) ensures that the first-best contract cannot be implemented; in particular, in trying to do so, the type- $L$ principal would try to mimic the type- $H$ principal. Thus, under this part, there is inefficiency in the least-cost-separating equilibrium. This holds, for example, if $\frac{\pi_{s}^{H}\left(a_{2}\right)}{\pi_{s}^{L}\left(a_{2}\right)}$ is not too large or if $u$ is linear.

I can now state the result.

Proposition 1 Suppose Assumption 1 holds. Then $\mathscr{P}^{S}$ and $\mathscr{M}^{S}$ intersect only at the $R S W$ (or the least-cost separating) equilibrium payoff. Suppose Assumption 2 also holds. Then, every $v \in \mathscr{M}^{S} \backslash\left\{\lambda \hat{v}^{H}+(1-\lambda) \hat{v}^{L}\right\}$ is strictly greater than every $z \in \mathscr{P}^{S}$ if and only if

$$
h\left(\bar{U}+a^{* L}-\frac{\left(a^{* L}-a_{1}\right) \pi_{s}^{L}\left(a_{2}\right)}{\pi_{s}^{L}\left(a_{2}\right)-\pi_{s}^{L}\left(a_{1}\right)}\right)>\underline{w} .
$$

Note that if $\underline{w}=-\infty$ then condition (1) is always satisfied.

Proof For the first statement, notice that, by definition, the best separating point-contract is the least-cost-separating point-contract; this gives the principal precisely the payoff of the RSW menu-contract, i.e. the lower-bound equilibrium menu-contract payoff. All other point-contracts will give the principal a (weakly) lower payoff and all other menu-contracts will give the principal a (weakly) higher payoff.

For sufficiency of the second statement, first suppose the $a^{* L}=a_{2}$. The left hand side of (1) is the wage that the type- $L$ principal pays to the agent in the public information benchmark if the agent fails: i.e. $w_{f}^{* L}:=h\left(\bar{U}+a_{2}-\frac{\left(a_{2}-a_{1}\right) \pi_{s}^{L}\left(a_{2}\right)}{\pi_{s}^{L}\left(a_{2}\right)-\pi_{s}^{L}\left(a_{1}\right)}\right)$ is the unique $w_{f}$ that is part of the solution to

$$
\begin{aligned}
\boldsymbol{\pi}^{L}\left(a_{2}\right) \cdot \mathbf{U}(\mathbf{w}) & =\bar{U}+a_{2} \\
\left(\boldsymbol{\pi}^{L}\left(a_{2}\right)-\boldsymbol{\pi}^{L}\left(a_{1}\right)\right) \cdot \mathbf{U}(\mathbf{w}) & =a_{2}-a_{1} .
\end{aligned}
$$


Recall that these equations are sufficient (given appropriately chosen $\mathbf{w}^{* H}$ ) for $\mathbf{w}^{* L}$ to be an equilibrium wage profile for the type $L$ principal since only the agent's downward incentive constraint will ever bind. By condition $(1), w_{f}^{*}(L)>\underline{w}$ (i.e. the solution is interior) and

$$
\Delta:=\frac{\lambda}{(1-\lambda)}\left[\boldsymbol{\pi}^{H}\left(a_{2}\right) \cdot \mathbf{U}\left(\hat{\mathbf{w}}^{\mathbf{H}}\right)-a_{2}-\bar{U}\right]>0
$$

by Assumption $2 .{ }^{14}$ Separation in the RSW equilibrium requires that the type- $H$ principal offer a contract that is more expensive than the first-best contract in order to dissuade the type- $L$ principal from mimicking her, thus generating a payoff higher than the reservation utility for the agent in the type- $H$ task. Since individual rationality only needs to be satisfied in expectation for menu-contracts, I can "transfer" these rents ceded to the agent from the principal of type- $H$ to the principal of type- $L$, essentially relaxing her individual rationality constraint by $\Delta$. The type- $L$ principal's menu-contract wage can therefore be solved as the unique solution to (3) and

$$
\boldsymbol{\pi}^{L}\left(a^{* L}\right) \cdot \mathbf{U}(\mathbf{w})=\bar{U}+a^{*}(L)-\Delta
$$

To show that the resulting set of wage profiles is cheaper for the principal, it is useful to use conditions (2), (3) and (4) to write $w_{f}$ as a function of $w_{s}$ we have

$$
\begin{aligned}
w_{f}^{L}\left(w_{s} ; A I C\right) & :=h\left(U\left(w_{s}\right)-\frac{a_{2}-a_{1}}{\left(\pi_{s}^{L}\left(a_{2}\right)-\pi_{s}^{L}\left(a_{1}\right)\right)}\right) \\
w_{f}^{L}\left(w_{s} ; I R\right) & :=h\left(\frac{\bar{U}+a_{2}-\pi_{s}^{L}\left(a_{2}\right) U\left(w_{s}\right)}{\pi_{f}^{L}\left(a_{2}\right)}\right) \\
w_{f}^{L}\left(w_{s} ; I R_{\Delta}\right) & :=h\left(\frac{\bar{U}+a_{2}-\Delta-\pi_{s}^{L}\left(a_{2}\right) U\left(w_{s}\right)}{\pi_{f}^{L}\left(a_{2}\right)}\right)
\end{aligned}
$$

Fix the type $H$ principal's contract at $\left(\hat{\mathbf{w}}^{H}, a_{2}\right)$. Note that the public information wage for the type- $L$ principal $\mathbf{w}^{* L}$ is defined such that $w_{f}^{L}\left(w_{s}^{* L} ; I R\right)=w_{f}^{L}\left(w_{s}^{* L} ; A I C\right)=w_{f}^{* L}$. Let $\tilde{\mathbf{w}}^{L}$

\footnotetext{
${ }^{14}$ The quantity $\Delta$ is chosen so that the type- $L$ principal extracts all of the agent's ex ante surplus.
} 
be the solution to $w_{f}^{L}\left(w_{s} ; I R_{\Delta}\right)=w_{f}^{L}\left(w_{s} ; A I C\right)$ if $\tilde{w}_{f}(L) \geq \underline{w}$ and $\tilde{\mathbf{w}}^{L}:=\left(\underline{w}, w_{f}^{L}\left(\underline{w} ; I R_{\Delta}\right)\right)$ otherwise. Since $w_{f}^{L}(\cdot ; A I C)$ is increasing ${ }^{15}$ and $w_{f}^{L}\left(\cdot, I R_{\Delta}\right)<w_{f}^{L}(\cdot, I R), \tilde{\mathbf{w}}^{L} \leq \mathbf{w}^{* L}$. Thus, $\tilde{\mathbf{w}}^{L}$ implements $a_{2}$ at a lower cost than $\mathbf{w}^{* L}$. If $\hat{v}^{H} \geq \boldsymbol{\pi}^{H}\left(a_{2}\right)\left(\mathbf{q}-\tilde{\mathbf{w}}^{\mathbf{L}}\right)$, then I am finished. Otherwise, choose $\tilde{\boldsymbol{w}}^{L}$ such that $\hat{v}^{H}=\boldsymbol{\pi}^{H}\left(a_{2}\right)\left(\mathbf{q}-\tilde{\mathbf{w}}^{\mathbf{L}}\right)$. This set of wage profiles is cheaper for the principal: by definition of $\hat{\boldsymbol{w}}^{H}$ and the fact that $\pi^{H}\left(a_{2}\right)>\pi^{L}\left(a_{2}\right), \tilde{w}_{s}^{L}<w_{s}^{* L}$ and $\tilde{w}_{f}^{L}<w_{f}^{* L}$. Thus, the principal is strictly better off ex ante. ${ }^{16}$

If $a^{* L}=a_{1}$, then $w_{f}^{* L}=w_{s}^{* L}=h\left(\bar{U}+a_{1}\right)$. Let $z=\min \left\{\bar{U}+a_{1}-\Delta, \underline{w}\right\}$. Then $\tilde{w}_{f}^{L}=$ $\tilde{w}_{s}^{L}=h(z)$ implements $a_{1}$ in menu-contracts. As before at $\tilde{\mathbf{w}}^{L}$, the constraint $P I C(L, H)$ is relaxed (since the type- $L$ principal now implements $a^{* L}$ at a lower cost and therefore receives a higher payoff) and therefore the type- $H$ principal can implement her effort at a lower cost. Thus, both types of the principal are strictly better off.

To show necessity, suppose that $w_{f}^{* L}=\underline{w}$. By definition, $w_{f}^{L}\left(w_{s}^{* L} ; A I C\right)=\underline{w}$. But then, since $w_{f}^{L}(\cdot ; A I C)$ is strictly increasing (see footnote 15 ), there is no $\tilde{w}_{s}$ that implements $a^{* L}$ such that $\tilde{w}_{s}<w_{s}^{* L}$, even for the relaxed individual rationality constraint. So the least cost contract that implements $a^{* L}$ remains $\left(w_{s}^{* L}, \underline{w}\right)$.

To see why sufficiency holds graphically, in the case where $a^{* L}=a_{2}$, observe Figure 1 . Note that the function $w_{f}^{i}\left(w_{s} ; A I C\right)$ represents the failure wage that makes the agent indifferent between exerting effort $a_{2}$ and effort $a_{1}$ if her success wage is $w_{s}$ and she knows that the principal is of type $i \in\{H, L\}$; to induce effort $a_{2}$, contracts must be to the right of this function in $\left(w_{s}, w_{f}\right)$-space. The function $w_{f}^{i}\left(w_{s} ; I R\right)$ represents the failure wage that makes the agent indifferent between participating and not if her success wage is $w_{s}$ and she knows the principal is of type $i \in\{H, L\}$; individually rational contracts are above this function in $\left(w_{s}, w_{f}\right)$-space.

The function $w_{f}^{i}\left(w_{s} ; I R_{\Delta}\right)$ is similarly the failure wage that makes the agent indifferent between participating and not if her success wage is $w_{s}$ and she knows the principal is of

${ }^{15}$ Note that $w_{f}^{L^{\prime}}\left(w_{s} ; A I C\right)=h^{\prime}\left(U\left(w_{s}\right)-\frac{a_{2}-a_{1}}{\left(\pi_{s}^{L}\left(a_{2}\right)-\pi_{s}^{L}\left(a_{1}\right)\right)}\right) U^{\prime}\left(w_{s}\right)>0$.

${ }^{16}$ One could also make both types of principal strictly better off. 


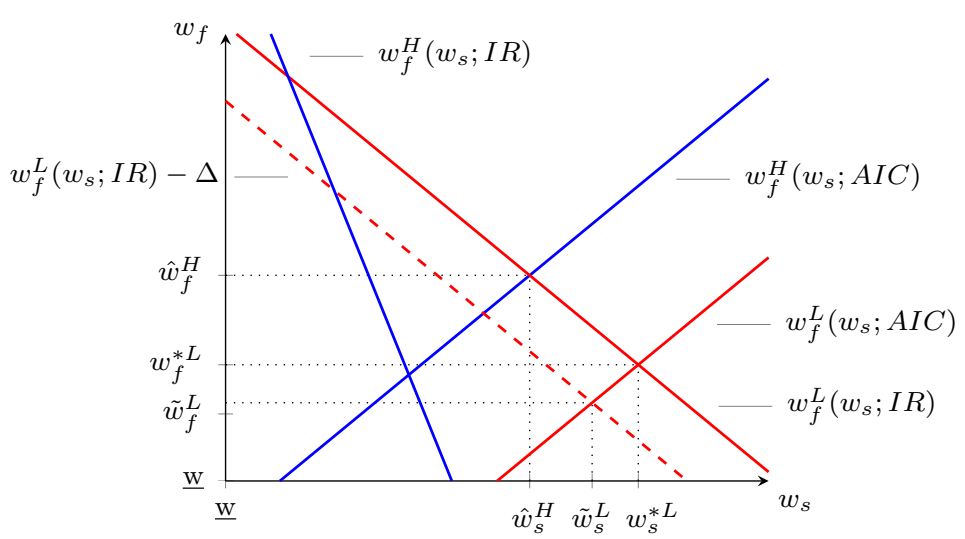

(a) Condition (1) is satisfied.

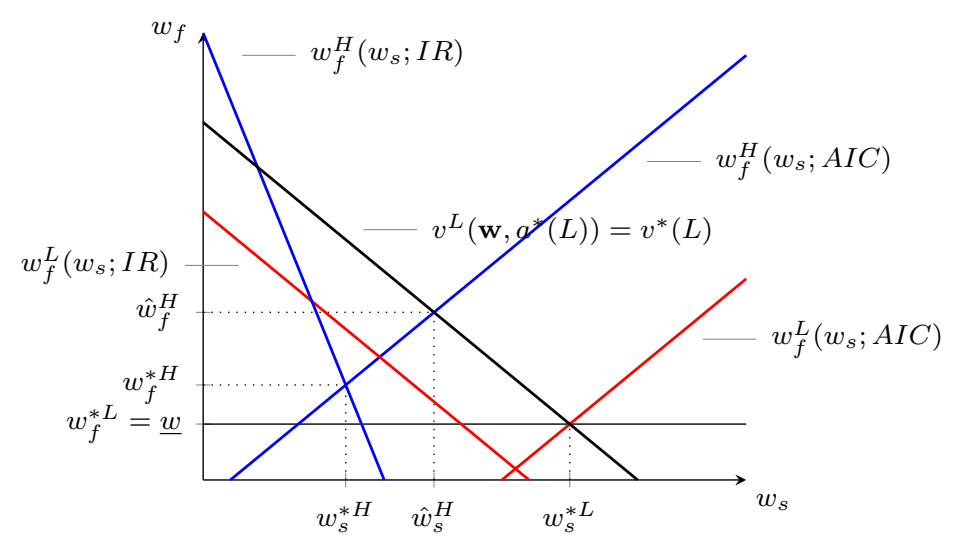

(b) Condition (1) is violated.

Figure 1: Illustration of Proposition 1.

type $i \in\{H, L\}$ and reservation utility is $\bar{U}-\Delta$. The individual rationality constraint for the type- $L$ principal effectively shifts down by some $\Delta>0$ (the red dashed line in Figure 1 (a)) making the less expensive contract $\tilde{\mathbf{w}}^{L}$ available to her. Thus, using menu-contracts, the principal can squeeze more rent from the agent by eliminating the slack in the agent's ex ante individual rationality constraint and transferring it across states in order to preserve her own incentive compatibility. This can be done so long as there are wage profiles that are less expensive than the RSW wage profile and are above $\underline{w}$ (i.e. condition (1) is satisfied). This is not an option using point-contracts since individual rationality must be satisfied ex post for separating contracts.

\section{Conclusion}

I have shown when allowing the principal to offer menu-contracts instead of point-contracts can increase the set of equilibrium payoffs and eliminate many poor equilibria for the principal. Additionally, I have characterized environments where equilibrium payoffs can be obtained using separating menu-contracts that are strictly higher than any separating equilibrium payoff using point-contracts. The results in this paper offer a useful guide to when the complexities of menu-contract equilibria can and cannot be safely ignored in moral hazard 
environments with an informed principal.

A natural question is whether menu-contracts are or can be used in practice. As noted by Segal and Whinston (2003), such contracts are rare but exist. They cite the example of a publishing company (the principal) retaining an option to cease publishing a book with the obligation to then return the copyright to the author (the agent). The hidden information of the publisher may be the stock of books competing with the author's for the publisher's promotional resources and attention: a book, regardless of the effort put into it, will more likely be a success if it is well promoted. The author decides whether to enter a contract with the publisher before learning her chances of success. Alternatively, any project-based employment offer that depends, at least implicitly, on the difficulty as well as the success of a project could be viewed as a menu-contract. As an example, return to the law firm introduced above. When hiring a new associate, a firm is unlikely to be precise about the type of cases that will be assigned but clear in stating (possibly implicitly) that rewards for more difficult cases are more highly powered than those of easy cases.

An important dimension of principal-agent relationships not explored here is repeated interaction. It is possible that a principal can use actions in a long-term relationship to signal the productivity of effort more cheaply than in the one-shot game with point-contracts. On the other hand, Beaudry (1994) demonstrates that in a two-shot game with point-contracts, such signalling is still costly, therefore leaving a role for menu-contracts to improve the principal's profits.

\section{A Appendix: Proofs}

Lemma 1 Suppose Assumption 1 holds. The RSW equilibrium is the least-cost separating equilibrium that has type-L principal offering $\left(\mathbf{w}^{* L}, a^{* L}\right)$ and the type-H principal offering 
the solution to

$$
I^{R S W}: \max _{\left(\mathbf{w}^{H}, a^{H}\right)} \boldsymbol{\pi}_{H}\left(a^{H}\right) \cdot\left(\mathbf{q}-\mathbf{w}^{H}\right) \quad \text { s.t. } \quad I R(1,0), A I C(1,0) \text { and } P I C(L, H) .
$$

The RSW contract always exists.

Proof Let $\left(\tilde{\mathbf{w}}^{H}, \tilde{a}^{H}\right)$ be a solution to $I^{R S W}$. First, I claim that the constraint $A I C(1,0)$ in problem $I^{R S W}$ must bind. Suppose $A I C(1,0)$ holds with strict inequality and let $\pi_{s}^{H} \geq \pi_{f}^{H}$. Then, decrease $w_{s}^{H}$ and increase $w_{f}^{H}$ slightly to $\left(\tilde{w}_{s}^{H}-\epsilon_{s}, \tilde{w}_{f}^{H}+\epsilon_{f}\right)$ for small $\epsilon_{s}, \epsilon_{f}>0$ so that $I R(1,0)$ and $A I C(1,0)$ still hold. Since $\pi_{s}^{H}>\pi_{s}^{L},\left(\epsilon_{s}, \epsilon_{f}\right)$ can be chosen such that the right hand side of $P I C(L, H)$ (possibly weakly) decreases while the objective function strictly increases. If $\pi_{s}^{H}<\pi_{f}^{H}$, increase $w_{s}^{H}$ and decrease $w_{f}^{H}$ slightly to $\left(\tilde{w}_{s}^{H}+\epsilon_{s}, \tilde{w}_{f}^{H}-\epsilon_{f}\right)$ for small $\epsilon_{s}, \epsilon_{f}>0$ so that $I R(1,0)$ and $A I C(1,0)$ still hold. Since $\pi_{s}^{H}>\pi_{s}^{L},\left(\epsilon_{s}, \epsilon_{f}\right)$ can again be chosen such that the right hand side of $P I C(L, H)$ (possibly weakly) decreases while the objective function strictly increases.

Second, I claim that $\left(\left(\tilde{\mathbf{w}}^{H}, \tilde{a}^{H}\right),\left(\mathbf{w}^{* L}, a^{* L}\right)\right)$ is incentive compatible. This is vacuously true for the type- $L$ principal since $P I C(L, H)$ is imposed in problem $I^{R S W}$ and $\mathbf{w}^{* L}$ is incentive compatible for the agent by construction. Further, $A I C(1,0)$ is imposed in problem $I^{R S W}$. It remains to show that

$$
\boldsymbol{\pi}^{H}\left(\tilde{a}^{H}\right) \cdot\left(\mathbf{q}-\tilde{\mathbf{w}}^{H}\right) \geq \boldsymbol{\pi}^{H}\left(a^{* L}\right) \cdot\left(\mathbf{q}-\mathbf{w}^{* L}\right) .
$$

I claim that (5) holds with strict inequality. Note that the curve in $\left(w_{s}, w_{f}\right)$ space implicitly defined by the agent's RSW incentive compatibility constraint for the type- $H$ principal,

$$
\left(\pi_{s}^{H}\left(\tilde{a}^{H}\right)-\pi_{s}^{H}\left(a_{1}\right)\right)\left[U\left(\tilde{w}_{s}^{H}\right)-U\left(\tilde{w}_{f}^{H}\right)\right]=\tilde{a}^{H}-a_{1},
$$


is strictly above that of the type- $L$ principal,

$$
\left(\pi_{S}^{L}\left(a^{* L}\right)-\pi_{S}^{L}\left(a_{1}\right)\right)\left[U\left(w_{s}^{* L}\right)-U\left(w_{f}^{* L}\right)\right]=a^{* L}-a_{1}
$$

due to Assumption 1. Further, the indifference curves of the type- $H$ principal are steeper than the type- $L$ principal's. Therefore, the indifference curves possess the single crossing property. If $\operatorname{PIC}(L, H)$ holds with equality, $\tilde{\mathbf{w}}^{H}$ lies to the north-west of $\mathbf{w}^{* L}$ in $\left(w_{s}, w_{f}\right)$ space which implies that (5) strictly holds. Otherwise, $\tilde{\mathbf{w}}^{H}=\mathbf{w}^{* H}$ and (5) strictly holds since $\pi_{s}^{H}\left(a^{* H}\right)>\pi_{s}^{L}\left(a^{* L}\right)$.

The RSW problem for the type- $H$ principal is more constrained than $I^{R S W}$ but $\left(\tilde{\mathbf{w}}^{H}, \tilde{a}^{H}\right)$ solves the latter problem and satisfies all the constraints of the former (with the type- $L$ contract specified as $\left.\left(\mathbf{w}^{* L}, a^{* L}\right)\right)$. Therefore it solves the RSW problem for the type- $H$ principal: $\left(\hat{\mathbf{w}}^{H}, \hat{a}^{H}\right)=\left(\tilde{\mathbf{w}}^{H}, \tilde{a}^{H}\right)$. Similarly, the RSW problem for the type- $L$ principal is more constrained than the public information problem, but $\left(\mathbf{w}^{* L}, a^{* L}\right)$ solves the latter problem and satisfies all the constraints of the former (with the type- $H$ contract specified as $\left.\left(\hat{\mathbf{w}}^{* H}, a^{* H}\right)\right)$. Therefore it solves the RSW problem for the type- $L$ principal.

To see that this menu-contract exists I first claim that $\mathbf{w}^{* L}(a)$ exists for any $a$. For $a=a_{1}$, $\mathbf{w}^{* L}(a)=\left(h\left(\bar{U}+a_{1}\right), h\left(\bar{U}+a_{1}\right)\right)$. For $a=a_{2}$, the constraints $\operatorname{AIC}(0,1)$ and $\operatorname{IR}\left(a_{2} ;\{0,1\}\right)$ will be satisfied with equality. Since $w_{f}^{L}(\cdot ; I R)$ is strictly decreasing and $w_{f}^{L}(\cdot ; A I C)$ is strictly increasing, they must intersect exactly once in $\mathbb{R}^{2}$. Denote this intersection point $\left(w_{s}^{\prime}, w_{f}^{\prime}\right)$. If $\left(w_{s}^{\prime}, w_{f}^{\prime}\right) \in[\underline{w}, \infty)^{2}$ I am done: $\mathbf{w}^{* L}=\left(w_{s}^{\prime}, w_{f}^{\prime}\right)$. Otherwise, the solution is $\mathbf{w}^{* L}=\left(w_{s}^{\prime \prime}, \underline{w}\right)$ where $w_{s}^{\prime \prime}$ satisfies $w_{f}^{L}\left(w_{s}^{\prime \prime}, A I C\right)=\underline{w}$. If the type- $L$ principal is indifferent between $a_{1}$ and $a_{2}$, set $a^{* L}=a_{2}$.

$I^{R S W}$ can be broken down into separate problems of minimizing the cost of implementing each effort then choosing most profitable effort. Note that $a^{* H}=a_{1}$ implies that $a^{* L}=a_{1}$ since the expected payoff from the agent's effort is strictly higher for they type- $H$ principal. Thus if $a^{* H}=a_{1}, \hat{\mathbf{w}}^{H}=\left(h\left(\bar{U}+a_{1}\right), h\left(\bar{U}+a_{1}\right)\right)$ which satisfies all the constraints of $I^{R S W}$ 
given our previous statement.

If $a^{* H}=a_{2}$ and $\operatorname{PIC}(L, H)$ does not bind, the solution to $I^{R S W}$ is simply $\mathbf{w}^{* H}$, which exists by an argument analogous to the previous one for the existence of $\mathbf{w}^{* L}$. Otherwise, the solution to $I^{R S W}$ is defined by

$$
\begin{aligned}
\left(\pi_{s}^{H}\left(a_{2}\right)-\pi_{s}^{H}\left(a_{1}\right)\right)\left(U\left(\hat{w}_{s}^{H}\right)-U\left(\hat{w}_{f}^{H}\right)\right) & =a_{2}-a_{1} \\
\boldsymbol{\pi}^{L}\left(a^{* L}\right) \cdot\left(\mathbf{q}-\mathbf{w}^{* L}\right) & =\boldsymbol{\pi}^{L}\left(a_{2}\right) \cdot\left(\mathbf{q}-\hat{\mathbf{w}}^{H}\right) .
\end{aligned}
$$

Equation (6) implicitly defines a strictly increase line in $\left(w_{s}, w_{f}\right)$-space while equation $(7)$ defines a strictly decreasing line in $\left(w_{s}, w_{f}\right)$-space. These lines therefore intersect exactly once in $\mathbb{R}^{2}$. Denote this intersection point $\left(w_{s}^{\prime}, w_{f}^{\prime}\right)$. If this $\left(w_{s}^{\prime}, w_{f}^{\prime}\right) \in[\underline{w}, \infty)^{2} \mathrm{I}$ am done: $\tilde{\mathbf{w}}^{H}=\left(w_{s}^{\prime}, w_{f}^{\prime}\right)$. Otherwise, the solution is $\mathbf{w}^{* L}=\left(w_{s}^{\prime \prime}, \underline{w}\right)$ where $w_{s}^{\prime \prime}$ satisfies equation (6) with $\hat{w}_{f}^{H}=\underline{w}$.

\section{References}

Beaudry, P. (1994). Why an informed principal may leave rents to an agent. International Economic Review, 35(4):821-832.

Chade, H. and Silvers, R. (2002). Informed principal, moral hazard, and the value of a more informative technology. Economics Letters, 74:291-300.

Inderst, R. (2001). Incentive schemes as a signaling device. Journal of Economic Behavior E Organization, 44(4):455-465.

Kaya, A. (2010). When does it pay to get informed? International Economic Review, $51(2): 533-551$.

Martimort, D., Poudou, J.-C., and Sand-Zantman, W. (2010). Contracting for an innovation under bilateral asymmetric information. The Journal of Industrial Economics, LVIII(2).

Maskin, E. and Tirole, J. (1990). The principal-agent relationship with an informed principal: the case of private values. Econometrica, 58(2):379-409.

Maskin, E. and Tirole, J. (1992). The principal-agent relationship with an informed principal, II: common values. Econometrica, 60(1):1-42. 
Myerson, R. B. (1983). Mechanism design by an informed principal. Econometrica, 51(6):1767-1797.

Rothschild, M. and Stiglitz, J. (1976). Equilibrium in competitive insurance markets: an essay on the economics of imperfect information. The Quarterly Journal of Economics, 90(4):629-649.

Segal, I. and Whinston, M. D. (2003). Robust predictions for bilateral contracting with externalities. Econometrica, 71(3):757-791.

Silvers, R. (2012). The value of information in a principal-agent model with moral hazard: the ex post contracting case. Games and Economic Behavior, 74(1):352-365.

Wagner, C., Mylovanov, T., and Tröger, T. (2015). Informed-principal problem with moral hazard, risk neutrality, and no limited liability. Journal of Economic Theory, 159:280-289.

Wilson, C. (1977). A model of insurance markets with incomplete information. Journal of Economic Theory, 16(2):167-207. 